\title{
A Residual Thermodynamic Analysis of Inert Wear and Attrition, Part 2: Applications
}

\author{
M. Gustavsson \\ Hot Disk AB, c/o Chalmers Science Park, Sven Hultins Gata 9, SE-41288 Gothenburg, Sweden \\ E-mail: Mattias.Gustavsson@ hotdiskinstruments.com
}

Received 03 November 2014, Revised 08 January 2015, Accepted 12 February 2015

\begin{abstract}
Some differential relations of inert wear- and attrition work mechanisms, derived in a parallel paper, are here applied. The consideration of relevant and suitable boundary conditions is called for. For ductile- and brittle-type target materials, one may often establish a direct- or proportional connection between the relevant sub-process work mechanism - referred to as wear work or attrition work - and corresponding net resulting wear- or attrition rates. Kinetic theory can be supportive in estimating model coefficients. In addition, additional thermodynamic considerations may be necessary, in order to estimate behavior at extremum conditions. The direct connection is demonstrated for the differential process of ductile wear, which encompasses most types of macroscopic-scale ductile erosion and 2-body- and 3-body ductile abrasion situations. The direct connection is also demonstrated for brittle erosion, and for impact wear. It is shown that the size effect on ductile erosion and abrasion - a hitherto not understood phenomenon - can also be explained by this connection.
\end{abstract}

Keywords: Boundary conditions; wear mechanisms; attrition mechanisms; particle size.

\section{Introduction}

The prospects of developing a less empirical theory of inert wear and attrition is investigated in [1].

A couple of different differential representations of some basic modes of inert wear and attrition were proposed in [1]. (Several other types of wear, described in the literature, are not covered in the following.) One topic in the present ancillary paper is to discuss ways in which these differential mechanisms may be used in practical applications. One application of interest is the implementation of these mechanisms in Computational Fluid Dynamics (CFD) flow solvers.

The overall modelling problem needs to be split up into two steps, where the first step represents the determination of the relevant flows and forces (that may often be computed in a CFD flow solver), while the second step represents the determination of breakage or deformation of the target material - utilizing the results of the Step 1calculation as model input, cf. Fig. 1. Cross-correlating interactions between Step 1 and Step 2 may occur (if the computation in Step 2 has impact on Step 1).

It should be noted that it is often not physically consistent to apply a traditional model (such as the Finnie model [2]), which in itself represents a combined (or "bundled") Step 1 and Step 2 computation, acting solely as a Step-2 model. Errors that follow from this have been discussed in Examples 1-2 in [1].

A differential Step 2-model of wear or attrition provides the following advantage: In contrast to a macroscopic traditional model, a differential Step 2-model need not account for macroscopic transient phenomena. The problem of modelling transient phenomena is a Step 1 modelling issue. [To illustrate, Finnie stated that his macroscopic single-particle erosion model fails to simulate the initial period in time when a dense-particle jet impacts a ductile target surface, a transient situation involving a kind of "splashing" impact behavior of the jet (possible to simulate in a CFD flow solver). Finnie found experimentally that considerably higher instantaneous erosion rates must occur during the initial period of a jet impact, in comparison to the erosion rates occurring at the following steady-state conditions. In [3] some simulations results are presented for a transient-bubbling fluidized bed, which indicate much higher erosion rates of internals at the initial stage of a bubble wake impact, when applying a differential ductile erosion model (proposed by [4]). In [5], also, much higher instantaneous erosion rates were modelled in the initial period of impact of a dense particle jet, supposedly in agreement with Finnie's observations.]

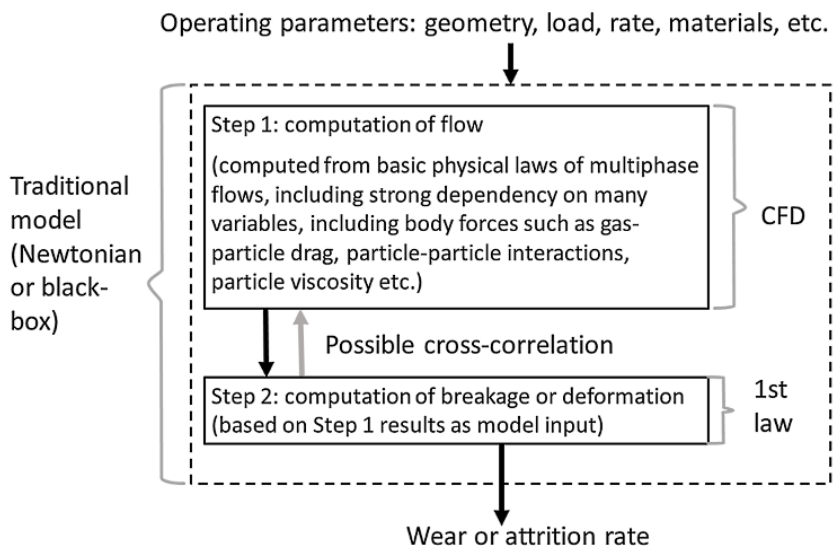

Figure 1. Breakup of modelling into two separate steps, referred to as Step 1 and Step 2. 


\section{Wear and Attrition Work}

An alternative theory is presented in [1], where new concepts wear work and attrition work are introduced derived utilizing a framework presented in [6]. It should be noted that wear- and attrition work represents anything from zero to a certain fraction of the total work. In many a situations, it appears that wear work is much smaller than total work.

Due to its definition, wear work is by default only weakly connected with total work. To illustrate: wear work may- or may not be proportional to total work. For instance, wear work is zero, when total work (and accompanying friction) is non-zero at process conditions below the wear onset threshold condition. In addition, the wear work is often difficult to quantify in magnitude when conducting experiments.

The analysis in this paper indicates that for a number of practical applications, an estimated wear work (or attrition work) expression appears to provide a proportional or a near-proportional correlation with net wear (or net attrition). A direct consequence of this observation is that due to the weak connection between wear work (or attrition work) and total work, the connection between total work and net wear (or net attrition) must be weak.

One key question in this paper is if there are any theoretical advantages of adopting the wear and attrition work concepts for practical applications? For instance, in order to derive models?

Also, due to the complexity in quantifying the wear and attrition work, another key question arises: what means does one have to analyze these residual- or sub-process work concepts?

A possible means to analyze the wear or attrition work is by considering the supposedly proportional connection between the sub-process work (i.e. wear or attrition work), and the corresponding sub-process outcome: wear $=C \times($ wear work $)$ or attrition $=C \times($ attrition work $)$. From these relations, a first approach would be to consider the hitherto proposed models available in the literature (for modelling net wear or net attrition) or published experimental results. [In Appendix A, the Archard model, applied for ductile abrasion, is compared with the Eulerian ductile wear work expression. In Appendix B, the singleparticle Finnie ductile erosion model is compared with the Eulerian ductile wear work expression.]

Another possible means to analyze the wear work or attrition work is to consider various ways of estimating the parameters incorporated in the basic Eulerian residual-work expressions presented in [1]. For instance, for the ductile wear expression involving parameters particle concentration, particle-phase viscosity, slip-flow coefficient, and flow gradients, all components which are possible to individually estimate. One way to proceed would be through dedicated experiments. Another way to proceed would be to consider extrapolating results obtained via the kinetic theory of gases relating to the behavior of these specific parameters. Kinetic theory has been successfully used in development of models for describing particle-phase- (or "granular"-) flows, including particleparticle- and particle-wall interactions, $c f$. relevant literature in the scientific field of multiphase flows. In Appendix C, the abrasive size effect in ductile wear processes is investigated with support from order-of-magnitude estimations obtained from kinetic theory.

\section{Conditions at Solid Surfaces \\ 3.1 Assumptions}

The assumptions for analytical treatment of processes occurring in fluid elements are partly that the particle flow - the assumed thermodynamic flow - can be represented by a continuum flow, following a fluidization assumption. This is assumed valid for a wide range of operating conditions, which incorporate also abrasive flow in small gaps in case of 3- and 2-body abrasion. Since the relevant irreversible residual processes are derived by a "self-comparison" procedure [6], the fundamental behavior of the derived basic wear and attrition processes are nevertheless possible to analyze as residual processes (i.e. non-linear, far-fromequilibrium, non-continuum processes), even though the thermodynamic flow is modelled as a continuum one.

However, in order to apply a continuum flow modelling, one needs to introduce models and make additional assumptions. In the following, the boundary-condition model Eq. (1) in [1] is applied in all situations involving the ductile wear process. This boundary condition is a good approximation, as it in principle allows the modelling of any averaged forces acting on the so-called gross contacting area of the target surface, for any type of averaged flow.

In the derivations below, adherence should be made to fundamental requirements that follow from thermodynamics, which for instance would be the requirement of bounded flow gradients, and non-zero fluid element volumes. Possibly, modifications of nominal models or introduction of new model assumptions may be required in order to adhere to fundamental requirements. Arguably, any modifications or assumptions made should not result in alteration of the basic structure of the wear or attrition mechanisms proposed.

The continuum- and fluidization assumption of the particle flow provides an ability to compute different types of averaged forces by means of fluid flow mechanisms (as calculated in CFD flow solvers) - for instance the computation of the averaged shear stresses acting across a gross contacting area. The feasibility of analyzing the averaged shear stresses acting across a gross contacting area (the surface boundary of the relevant fluid elements) should be considered.

A relevant question to pose, in this respect, is whether the need for known conditions occurring on net contacting areas is required at all? Surveying the literature, it appears that ductile abrasion tests (including those reported by Archard) are performed with a scratcher of non-zero contact area. The discussion in the literature states that the real forces acting on individual contacting/interacting/ploughing tips in abrasion experiments are not known. Also, regarding ductile erosion, Finnie claimed that the real forces acting on the cutting tips in ductile erosion experiments were not known - Finnie's derivation only assumed that the ratio of normal to tangential forces acting on the tip were constant throughout the cutting process.

Lacking other alternatives, the analysis of averaged forces acting across gross contacting areas, should to the present view be validated by comparing present outcome with corresponding experimental results.

\subsection{Ductile Wear}

The theoretically-derived Eq. (30) in [1], gives for the case of ductile wear: 


$$
\begin{aligned}
& \dot{W}_{\text {ductile wear }}+\underbrace{\dot{W}_{\text {transfer/reflection }}+\dot{W}_{\text {friction }}+\dot{W}_{\text {etc. }}}_{\dot{W}_{\text {other,irr }}}= \\
& =\underbrace{\int_{\text {S, wall }} \alpha_{p}\left(\tau_{p, 21, \text { proc }}-\tau_{p, 21, \text { noproc }}\right) U_{p 1} \mathrm{~d} A}_{\propto \text { ductileerosionor abrasion(term (c)) }} \\
& =\int_{\mathrm{CS}, \text { wall }} \alpha_{p}\left(\mu_{p, \text { proc }}-\mu_{p, \text { noproc }}\right) \frac{\partial U_{p 1}}{\partial y_{2}} L \frac{\partial U_{p 1}}{\partial y_{2}} \mathrm{~d} A \\
& =\int_{\mathrm{CS}, \text { wall }} L\left(\left.\delta_{\mathrm{SCVD}, \text { res }}\right|_{\text {surface }}\right) \mathrm{d} A
\end{aligned}
$$

(Eq. (1a) is expressed in units W.) In Eq. (1a), we have incorporated the mathematical function $\left.\delta_{\mathrm{SCVD}, \text { res }}\right|_{\text {surface }}$ (units $\mathrm{W} / \mathrm{m}^{3}$ ) - referred to as the residual shearing components of the viscous dissipation, $c f$. mathematical definition [5] - to simplify notation. (It should be noted that for ductile abrasion all terms inside this function - extracted at the wall - are zero except terms containing $\left(\partial U_{p 1} / \partial y_{2}\right)^{2}$, and the same is practically also the case for ductile erosion.)

The literature has limited information on the behavior of $L$, unless dealing with non-erosive gas molecular-solidwall impacts ( $c f$. kinetic theory).

A dense-particle-flow ductile erosion model was earlier proposed in [5]:

ductile erosion rate $=\left.C_{3} \delta_{\mathrm{SCVD}}\right|_{\text {surface }}$

This empirical model was proposed based on the exclusion of apparently irrelevant terms in nominal total work of surface forces components [5]. Although this model is based on fundamentally different assumptions, there are direct mathematical similarities between Eq. (2a) and Eq. (1a) that make an association with current theory of interest.

The differences between the empirical model proposed in [5] and extant theory, can be summarized as follows:

(a) The particle flows are principally different in present theory, than those assumed in [5], $c f$. Section 2.3 in [1].

(b) The energy balances accounted for in present theory cannot be accurately accounted for in a nominal nonerosion or non-attrition particle-flow solver as utilized by [5] when applying Eq. (2a).

(c) Threshold phenomena are not naturally accounted for in the model proposed in [5].

(d) The mathematical difference between the empirical model Eq. (2a) and a corresponding theoretically-derived expression based on wear work [cf. e.g. Eq. (2b) discussed below], if identical particle flows can be assumed, involves the composition of the model constant. For instance, Eq. (2a) would then be mathematically identical to Eq. (2b) if:

$$
C_{3}=K^{\text {strong }} L\left(\mu_{p, \text { proc }}-\mu_{p, \text { noproc }}\right) / H \chi \mu_{p}
$$

Despite principal differences, in situations where particle-flow behavior can be assumed to be practically indifferent to whether erosion (or attrition) is present or not, the implications are that the theoretically-derived expressions of the present work would yield similar denseparticle flow erosion simulations as demonstrated in [5] the only difference being a model constant.
Reformulating the theoretically-derived expression Eq. (1a) into units $\mathrm{W} / \mathrm{m}^{2}$ one obtains:

$$
\begin{aligned}
& \dot{w_{\text {ductilewear }}}+\underbrace{\stackrel{\bullet}{w_{\text {transfer/reflection }}+\dot{w}_{\text {friction }}+\dot{w}_{\text {etc }}}}_{\dot{w}_{\text {other }, \text { irr }}}= \\
& =\left.L \delta_{\mathrm{SCVD}, \text { res }}\right|_{\text {surface }} \\
& \stackrel{\bullet}{w_{\text {ductilewear }}} \propto L \delta_{\mathrm{SCVD}} \text {, res }\left.\right|_{\text {surface }}
\end{aligned}
$$

Assuming a directly proportional correlation between wear work and wear rate, one can set:

$$
\frac{\text { wear work rate }}{\text { wear rate }}=C_{2} \text { (constant) }
$$

The local wear work rate, following Eq. (1c), may be expressed as:

$$
\dot{w}_{\text {ductilewear }}=\left.C_{1} L \delta_{\mathrm{SCVD}, \text { res }}\right|_{\text {surface }}
$$

In order to assess the applicability of the theoreticallyderived relation Eq. (1d) for ductile wear situations (dense particle-flow ductile erosion is already partly covered for by results in [5]), it is necessary to validate Eq. (1d) for other ductile wear situations.

In Appendix A the empirical Archard model is reformulated into an Eulerian expression, based on total work. Comparing the Eq. (1d) with the reformulated Archard relation (applied for ductile abrasion), provides a proportional connection between the Archard relation and the introduced concept of wear work.

Hence, when introducing the proportionality $K^{\text {strong }} / H \chi=C_{1} / C_{2}$, cf. Appendix A, one obtains a theoretically-derived expression which is capable of reproducing Archard's model (applied for ductile abrasion):

ductile wear rate $=\left.\frac{K^{\text {strong }} L}{H \chi} \delta_{\mathrm{SCVD} \text {, res }}\right|_{\text {surface }}$

In Appendix B, it is argued that Eq. (2b) can also reproduce the Finnie single-particle ductile erosion model.

\subsection{Influence of Particle Size on Ductile Wear and Ductile Attrition}

Several experiments show the erosion or abrasion rate as strongly related to the particle size [7], cf. Example 1. [The particle-size influence was earlier described [8] as "perhaps the single most puzzling aspect of the erosion and abrasion of ductile materials".]

In order to investigate the particle-size influence, it is to the present author's view important to investigate the particle-slip behavior accounted for through the slip-flow coefficient $L$, as well as the coefficient $\mu_{p \text {,proc }}-\mu_{p \text {,noproc }}$ incorporated in Eq. (1d) (within the term $\delta_{\mathrm{SCVD}}$, res $)$.

From the $1^{\text {st }}$ law of thermodynamics, one obtains for both erosive and abrasive flows: 
STATEMENT (A): The fundamental reason allowing for -

an irreversible shear work transfer $\dot{w}_{\text {shear,surface, irr }}$ at the target surface is due to a slip-flow mechanism. Simply stated, if there is no slip-flow mechanism at the target

surface, an irreversible shear-work transfer $\dot{w}_{\text {shear,surface, irr }}$ cannot occur.

The slip coefficient $L$ may differ between erosive and abrasive flows. In the nominal partial-slip boundary condition [9], $c f$. Eq. (1) in [1]:

$\mathbf{U}_{p, \mathrm{t}}=L \frac{\partial \mathbf{U}_{p, \mathrm{t}}}{\partial \mathbf{n}}$

the slip coefficient:

$$
L_{\mathrm{nom}}=\varphi d_{p} / 6 \alpha_{p} \sqrt{2}
$$

represents the mean-free path of the particles [10], in case the particles interaction with the wall can be characterized as a "diffuse reflection". The present author believes that setting $L \approx L_{\text {nom }}$ following Eq. (6) gives a fairly good approximation for dense erosive flows (i.e. flows with strong particle-particle interaction).

It is here argued that setting $L \approx L_{\text {nom }}$ according to Eq. (6) is principally correct for 3-body abrasive flows. For 2-body abrasive flows, one may assume $L=L_{\text {nom }}=\Phi$, where $\Phi$ represents the average gap width.

It follows from Eq. (6) that $L \propto d_{p}$. (For 2-body abrasive flows, $L \cong d_{p}$. .) Also, from kinetic theory, for erosive flows it follows that the coefficient $\left(\mu_{p, \text { proc }}-\mu_{p, \text { noproc }}\right) \propto L$ (since $\left.\mu_{p} \propto L\right)$, which in turn gives $\left(\mu_{p, \text { proc }}-\mu_{p \text {, noproc }}\right) \propto d_{p}$. In sum, one may from Eq. (1c) - after expansion and re-grouping - state that:

$\dot{w}_{\text {ductilewear }} \propto d_{p}{ }^{2} \times\{\text { flow gradients }\}^{2}$

where the flow gradients are bounded in the vicinity of the target surface. Equation (1e) is applied in Appendix C to analyze the size effect of ductile wear.

Note: For the curves correlating the wear work with the inert net wear rate, for different particle sizes, assume both parameters $C_{1}$ and $C_{2}$ to be strictly constant (independent of particle size). If parameter $\chi$ is assumed to not vary with the particle size, $d_{p}$, then the coefficient $K^{\text {strong }}$ will become strictly constant (independent of particle diameter, independent of wear work, as well as independent on external total work). In contrast, if instead connecting the original Archard relation to the experimental data, and thereby extracting $K^{\text {weak }}$, one finds immediately that the coefficient $K^{\text {weak }}$ displays a strong dependency on particle size, as well as other experimental parameters, i.e.:

$$
K^{\text {weak }}=K^{\text {weak }}\left(d_{p}, \dot{W}_{\text {total }}, \varepsilon, \ldots\right)
$$

For particle-surface attrition, literature results indicate decreasing attrition rates with decreasing particle diameter. This immediately follows at least for particles subject to ductile attrition: Equation (5) provides an invariant representation of the flow velocity which may be applied in Eq. (34) in [1] in the vicinity of a solid surface. A corresponding analysis gives:

$\left.\dot{w_{\text {ductileattrition }} \propto-d_{p} \times\{\text { flow gradients }}\right\}^{2}$

for dense flows, a relation having significant implications for the analysis of attrition, $c f . e . g$. Section 3.4.

Returning to the issue of the approximate relation $L \approx L_{\text {nom }}$ following Eqs. (5)-(6), however, there are a couple of issues which may alter this picture:

First, any type of target-surface wear or particle-surface attrition as a result of particle interaction with the target surface will - to a lesser or greater degree - distort the assumed conditions for the model in Eq. (6).

Secondly, the following observations can be made from ductile erosion experiments:

OBSERVATION (A): For an erosive flow (singleparticle impact or an impinging jet flow) approaching $90^{\circ}$ impact angle, where $\mathbf{U}_{p, \mathrm{t}} \rightarrow \mathbf{0}$, experimental results indicate that $L \rightarrow 0$.

OBSERVATION (B): A free-slip flow, used in modelling of potential flows (assuming zero viscosity of the fluid medium) where the boundary condition is set to $\frac{\partial \mathbf{U}_{p, \mathrm{t}}}{\partial \mathbf{n}}=\mathbf{0}$ but $\mathbf{U}_{p, \mathrm{t}} \neq \mathbf{0}$, requires $L \rightarrow \infty$. [In this case, no shear worktransfer can occur at the target surface, neither reversible $\stackrel{\bullet}{\left.\boldsymbol{w}_{\text {shaft, rev }}\right)}$ nor irreversible $\left(\stackrel{\bullet}{\left.\left.\boldsymbol{w}_{\text {shear, surface, irr }}\right) .\right]}\right.$

In experiments [11] it was found that the erosion ripple phenomena occurred with strongest erosion rate at low angles of macroscopic jet impact. However, the erosion ripple phenomena apparently ceased to occur when the angle of impact was further reduced, and approached near zero (i.e. when the jet flow was almost parallel to the target surface). This latter condition resembles a free-slip condition where $L \rightarrow \infty$, i.e. where erosion cannot occur (according to Observation (B)).

EXAMPLE 1: For single-phase gas- or liquid fluid flows in traditional fluid dynamics, the mean-free path $L$ is so small that it is normally neglected at solid boundaries, i.e. Eq. (5) is replaced with a so-called no-slip boundary condition assumption, $\mathbf{U}=\mathbf{0}$, which directly amounts into $\dot{W}_{\text {surface,irr }}=0$, according to Statement (A). Hence, ductile erosion or abrasion cannot occur. It is widely accepted in the field of wear that general single-phase gaseous or liquid flows do not generate ductile erosion. However, the wear literature often attributes this result to a matter of "hardness of the abrasives", where it is stated that the abrasive or erosive particles must inhibit a hardness greater than that of the target surface in order for ductile erosion to occur. While this condition applies for the majority of erosion and abrasion processes, it does not apply for SIMS (Secondary Ion Mass Spectrometry) experiments: Ions - which do not inhibit any hardness at all - do create ductile erosion, since irreversible shear-work transfer can be obtained (above a certain threshold) when the specific ion-bombarding 
conditions are applied. The slip-flow in the vicinity of the target surface must exist for erosion to occur in SIMS experiments, and the combination of a strong particleparticle interaction in the vicinity of the target surface, together with a slip-flow along the target surface, will eventually generate a slip-roll mechanism, resulting in the formation of erosion ripples on the target surface, $c f$. wavelength analysis in [6].

\subsection{Ductile and Brittle Attrition in a TMP Wood Chip Refiner}

A TMP refiner [12-15] is a grinder, mechanically processing wood chips into paper fibers. At the inlet, wood chips are fed to the grinder, which are then processed mechanically while water is injected at the inlet. Saturated steam exits from both inlet and outlet. At the outlet, paper fibers are ejected. The gap width $\Phi=\Phi(r)$, a parameter critical for the refining process, is large at the inlet and decreases towards the rim of the refiner, typically of the order $\Phi \propto r^{-x}$, where $x \geq 1$. Two distinct zones exist: a coarse pattern zone at lower radii, and a fine pattern zone towards the rim of the refiner. Most attrition work (per unit area) is obtained in the fine pattern zone, approaching a maximum work towards the rim of the refiner, i.e. at the exit zone. The refiner is run continuously over a timeperiod of 7 weeks - the time it takes to wear down the refiner plates.

The stationary 1D flow conditions versus radius can be modelled [12], assuming circular symmetry - for the three involved phases saturated steam, saturated water and dry wood. The following expression:

$$
\stackrel{\bullet}{w_{\text {res, av }}}=\stackrel{\bullet}{w_{\text {proc }, \text { av }}-} \stackrel{\bullet}{w_{\text {noproc }, \text { av }}}
$$

was empirically proposed [12] as a model for averaged "defibration" work (here referred to as attrition work) at radial position $r$. The process work $\dot{w}_{\text {proc,av }}$ (referred to as "total" work in [12]) was experimentally determined through recording of averaged shear stress versus radius (a refiner operates at typically 3.000 or 3.600 r.p.m. with net total work consumption around $10 \mathrm{MW}$ ). Heat is negligible in this process. The corresponding average no-process work •

$w_{\text {noproc,av }}$ (referred to as "thermodynamic" work in [12]) was experimentally determined through recording of temperature versus radius, assuming dry wood acting as an incompressible pure substance [16], without considering energy of surfaces or -deformation for the dry wood particles (or for the metal bars of the grinding disks, subject to abrasion wear). The mathematical difference between process work and no-process work (for identical thermodynamic flows) gives the defibration work $\dot{w}_{\text {defibration }}=\dot{w}_{\text {res, av }}$ versus radius, $c f$. Fig. 2, i.e. the net particle attrition work (including plate wear work) at radial position $r$. Implementing balance relations for flow for the three phases, in terms of mass balance (continuity), energy balance ( $1^{\text {st }}$ law of thermodynamics), and entropy balance ( $2^{\text {nd }}$ law of thermodynamics) for the open control volume represented by the slice $r$ to $r+\mathrm{d} r$ and local minimum gap width $\Phi(r)$, and one additional relation to close the set of equations [12], the radial flow of the three phases vs. radius can be determined rather accurately [the simulated "consistency" (a measure of amount of saturated water remaining inside exiting fibers) agreed within typically 1 $2 \%$ compared to corresponding experiments, also at timevarying operating conditions.]

The relation Eq. (9), which provides net total defibration work starting at typically $15 \%$ (25\% according to Fig. 2 and [13]) of total work input to the grinder, and gradually decreasing to typically around 5\% (10\% according to [13]) towards the end of the life-time (approximately 7 weeks) of the refiner plates, due to wear-down of the same [14-15]. This indicates that only a small fraction of total work input to the TMP refiner actually results in attrition work. A consequence of the wear-down process is that the originally sharp bars tend to round, resulting in a gradual decrease in the overall frictional performance of the plates (in the rotational direction), $c f$. Fig. 3. To compensate for this, the normal pressure applied on the refiner disks is gradually increased to maintain an overall time-averaged frictionalshearing work. When increasing the normal pressure the gap width gradually decreases - a decrease which has drastic impact on $\Phi \propto r^{-x}$, in particular in the fine pattern zone near the rim of the discs. In effect, the gap width decrease can be correlated with an increase in the variable $x$. Eventually, when the plates are fully worn out, plate clash occurs.

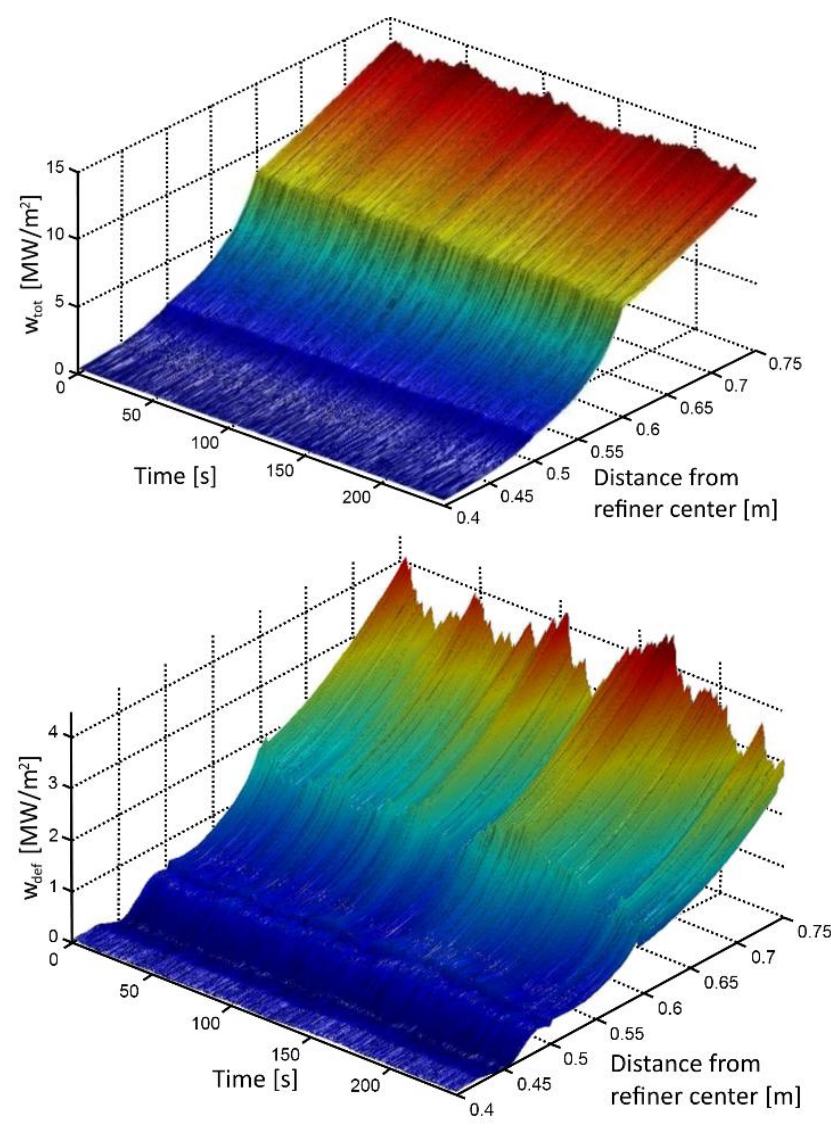

Figure 2. Total work (top) and defibration work (bottom), cf. [13].

Experiments indicate that attrition occurs only on few occasions, and at a few positions, $c f$. Fig. A3 in [6]. Monitoring a particle trajectory, one finds that a particle circles the grinder approximately 10 rotations, from inlet to outlet, which means that a particle passes approximately 2000-4000 bars in the grinding process (depending on the grinder geometry and plate gap). Since dust is not generated in this process - which would be the case if bar passage 
contributed to a so-called "erosion attrition" mechanism (erosion of particle surfaces) - it is reasonable to assume that a particle breakage will break a typical particle into two, each with a diameter of between $50 / 50 \%$ or $80 / 20 \%$ of the original particle - a geometric average of $63 \%$. In each dimension, to break down a particle from initial dimensions of $25 \mathrm{~mm}$ × $20 \mathrm{~mm}$ x $4 \mathrm{~mm}$ (a typical wood chip entering the inlet), to a paper fiber with a length of $5 \mathrm{~mm}$, width 0.5 $\mathrm{mm}$, and height $0.5 \mathrm{~mm}$ (at the outlet), requires approximately $4 \times 8 \times 4.5=144$ breakages, i.e. a breakage rate of approximately $3-8 \%$. In effect, breakage or irreversible deformation occurs at every $13^{\text {th }}$ to $30^{\text {th }}$ bar passage, a fairly low rate, and confirms the notion of attrition only occurring at spot-wise locations. If multiple breakages occur at the same bar passage, for instance when flocculation patterns form [6], the zones of attrition occupy an even smaller area fraction of the total TMP refining zone cross-sectional area. The attrition process in a TMP refiner is hence concluded not to be a continuous one.

If the attrition process occurs in a non-continuous manner, one may assume that it does so in small zones. Is it possible to estimate the local forces and stresses occurring in these?

To analyze this, consider the average experimentallyrecorded shear stress, in the rotational direction, across a refiner disk versus radius. Experiments indicate $\tau_{\mathrm{av}} \propto r$.

Furthermore $\dot{w}_{\text {proc, av }}=\tau_{\mathrm{av}} \times U_{r}=\tau_{\mathrm{av}} \times \omega r \propto r^{2}$, where $U_{\mathrm{r}}$ is the local rotational velocity at radial position $r$, and $\omega$ is the angular velocity [radians/sec].

For ductile attrition, Eq. (8) indicates flow gradients, i.e. local stresses required to break particles having a diameter $d_{\mathrm{p}}$, requires:

$$
\begin{aligned}
& \left(\dot{w_{\text {ductileattrition,irr }}}\right)_{\mathrm{av}}= \\
& =\frac{1}{V_{\mathrm{CV}}} \int_{\mathrm{CV}}\left(\dot{w}_{\text {ductileattrition,irr }}\right)_{\text {break.spos }} \mathrm{d} V \\
& \propto \sum \frac{1}{V_{\mathrm{CV}, \text { spots }}} \int_{\mathrm{CV} \text {,spots }}\left(d_{\mathrm{p}} \times\{\text { flow gradients }\}^{2}\right) \mathrm{d} V
\end{aligned}
$$

Since:

$$
\left(\dot{w_{\text {attrition,irr }}}\right)_{\mathrm{av}} \propto r^{2}
$$

(regardless of attrition being ductile or brittle, a valid relation throughout the life-time of the disk plates [12]), one may, based on estimation of flow gradients - incorporating

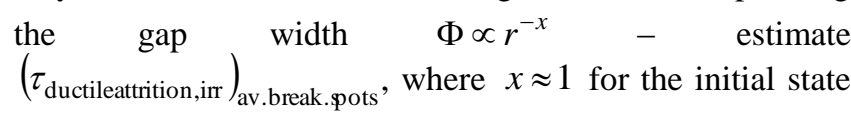
of the refiner (when ductile attrition is dominant) and $x>1$ when the pressure over the refiner disks is increased resulting in a smaller plate gap towards the rim of the refiner (when brittle attrition increases while ductile attrition decreases).

For ductile attrition in a TMP refiner, experiments indicate that the wood chips and paper fibers are fairly evenly-distributed across the refiner disc, at least initially, $c f$. Fig. A3 in [6].
As the refiner plates are gradually worn down, the net attrition work is continually decreased. However, as the net ductile attrition is significantly reduced, the net brittle attrition increases. Gradually, flocculation patterns, starting with several modes, emerge. Initially, these are hard to observe experimentally. However, when the number of modes gradually reduce, they become more dominant in experiments, $c f$. Fig. A3 in [6]. In these flocculation patterns, brittle attrition will occur. Towards the end of the life-time of the refiner plates, essentially only brittle attrition will occur: The stage at which 3-mode flocculation pertains, will after further wear-down of the refiner plates result in a 2-mode flocculation pattern, requiring yet again a normal pressure increase, $c f$. [6]. Soon, toward the end of the plates' life-time, any additional wear-down and further pressure increase beyond this point will cause plate clash, i.e. the TMP grinding process must be stopped, and the plates replaced by new ones.

Regarding forces at play within a bundled flocculation pattern, where brittle attrition occurs, considering the comparatively small volume occupied by the flocculation pattern, together with a reduced friction force (due to the wear down-, smoothening- and polishing of the refiner bars), an order-of-magnitude modelling would find that large particle-particle pressures (and corresponding large pressure gradients) exists within the flocculation zone amounting to brittle attrition. [The ease at which wood chips/fibers mixture apparently slip across polished bars at the final stages of the worn-down TMP refiner plates lifetime is sometimes depicted as a "soap-like" behavior of the multiphase pulp-fiber mixture, among scientists working on TMP wood chip refiners.]

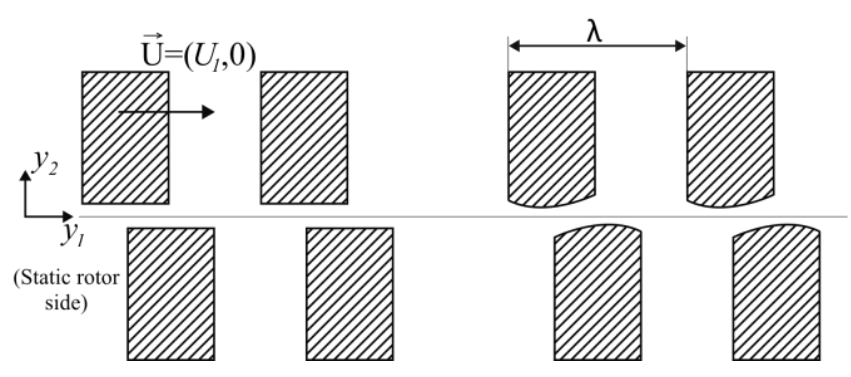

Figure 3. Attrition in a TMP wood chip refiner in the fine pattern zone, near the rim, can be characterized as ductile attrition when refiner bars are sharp (left). The abrasive ductile wear rate along the bar surface (in the rotational direction) is highest at the front edge, and lowest in the center-rear region. The rear edge has a higher abrasion rate than the center-rear region. (Experiments on normal-and shear forces vs. time are available in the Pulp \& Paper literature, which provide a Lagrangian picture of force variations during bar passage. These experiments confirm that normal-and shear forces attain a maximum at front edge passage.) Later, when the refiner bars are worn out (right), overall abrasion is similar across the refiner bar. At this stage, brittle attrition will occur. A gradual change in attrition behavior can be observed, from the state of fresh plates (left) to the state of worn-out plates (right), by studying the statistical particle-size distribution of the produced fibers [6].

\subsection{Deformation Wear}

A special case of irreversible viscous work transfer is illustrated in Fig. 4 in [1]. Here dissipation is caused by the normal work components of the stress tensor (not requiring a slip flow) which may result in deformation wear in the form of brittle erosion (for brittle-type solids) or workhardening (of ductile-type solids). If so, an irreversible work transfer to the target surface - through a normaldirection momentum exchange on the particle scale - takes 
place, in conjunction with exhaustion of particles' kinetic energy.

The irreversible work transfer for normal-direction momentum exchange (also on the particle scale) is proportional to the irreversible work from the specific components of term (c)-type wear in Eq. (30) in [1] which relates to indentation according to Fig. 4 in [1]. For the following analysis one may assume the bulk viscosity of the particle phase $\xi_{\mathrm{p}}=0$. For an indenting tip which may irreversibly indent the target surface through a momentum exchange, it is in turn reasonable to assume $\partial_{1} U_{1} \cong 0$ at the boundary. Also, for an irreversibly indenting tip of a particle, a crude analysis (in the vicinity of the target surface) of the velocity reduction [from pre-impact speed $U_{2}=-\left|U_{\text {impact }}\right|$ at the instant the tip of the particle touches the target surface, to speed zero when the tip has irreversibly penetrated the target surface a short distance $\Delta y_{2}=-\left|\Delta_{\text {indent }}\right|$, an indentation or penetration distance which can be assumed to have a small dependency on $U_{2}=-\left|U_{\text {impact }}\right|$, say $\Delta y_{2}\left(U_{2}\right) \propto\left|U_{2}\right|^{n}$, where $1 \gg n \geq 0$ ] results in a crude approximation $\partial_{2} U_{2} \approx Z_{2} U_{2}{ }^{1-n}$ at the boundary, where $Z_{2}>0$ depends on the average sharpness and/or sphericity of the indenting particles, as well as on the hardness of the target surface. From this, one may extract a boundary condition: $U_{2} \approx\left(\partial_{2} U_{2} / Z_{2}\right)^{1 /(1-n)}$ in the vicinity of the target surface.

Hence, from these approximations and assumptions, when applied in the theoretically-derived expression Eq. (30) in [1], gives:

$$
\begin{aligned}
& \dot{W}_{\text {deformation wear }}+\underbrace{\dot{W}_{\text {transfer/reflection }}+\dot{W}_{\text {friction }}+\dot{W}_{\text {etc. }}}_{\dot{W}_{\text {other,irr }}}= \\
& \underset{\substack{\uparrow}}{\approx} \frac{1}{Z_{\mathrm{p} \mathrm{CS}, \text { wall }}} \int_{\text {in thiscase }}\left(\left.\delta_{\mathrm{NCVD}, \text { res }}\right|_{\text {surface }}\right)^{(2-\mathrm{n}) / 2(1-n)} \mathrm{d} A
\end{aligned}
$$

where $Z_{\mathrm{p}}>0$ in addition to average sharpness and/or sphericity of the indenting particles and hardness of the target, also incorporates a small dependency on components $\alpha_{\mathrm{p}}$ and $\mu_{\mathrm{p}, \mathrm{proc}}-\mu_{\mathrm{p}, \text { noproc }}$.

In Eq. (12a), we have incorporated the mathematical function $\left.\delta_{\mathrm{NCVD}, \text { res }}\right|_{\text {surface }}\left(\right.$ units $\mathrm{W} / \mathrm{m}^{3}$ ) - referred to as the residual normal components of the viscous dissipation, $c f$. mathematical definition [5] - to simplify notation. (It should be noted that all terms inside $\left.\delta_{\mathrm{NCVD}, \text { res }}\right|_{\text {surface }}$ in Eq. (12a) - extracted at the wall - are zero, except terms containing $\left(\partial U_{2} / \partial y_{2}\right)^{2}$.) (Equation (12a) is expressed in units W.) The local action of Eq. (12a) in units $\mathrm{W} / \mathrm{m}^{2}$ :

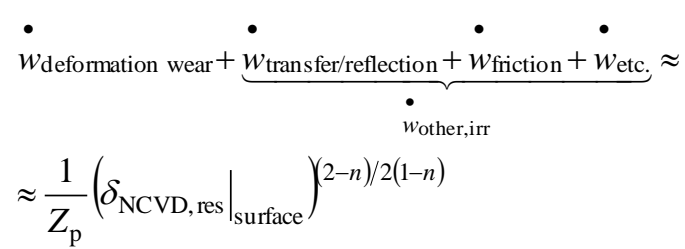

or

$$
\begin{aligned}
& \dot{w}_{\text {deformation wear }} \propto \frac{1}{Z_{\mathrm{p}}}\left(\left.\delta_{\mathrm{NCVD}, \text { res }}\right|_{\text {surface }}\right)^{(2-n) / 2(1-n)} \\
& =\left.\frac{\left(\frac{4}{3} \alpha_{\mathrm{p}}\left(\mu_{\mathrm{p}, \mathrm{proc}}-\mu_{\mathrm{p}, \text { no proc }}\right)\left(\partial_{2} U_{2}\right)^{2}\right)^{(2-n) / 2(1-n)}}{Z_{\mathrm{p}}}\right|_{\text {surface }}
\end{aligned}
$$

In [5] it was demonstrated that the empirically-proposed (and mathematically similar) expression $\left.C \cdot \delta_{\mathrm{NCVD}}\right|_{\text {surface }}$ can approximately model deformation wear.

In case $n \approx 0$, and for similar particle flows, one will find that the practical difference between the theoreticallyderived expression Eq. (12c) and the empirically-proposed model $\left.C \cdot \delta_{\mathrm{NCVD}}\right|_{\text {surface }}$ is only a model constant.

Fundamental differences exist on different modelling assumptions made in [5] as compared to modelling assumptions made in the present work. These are listed as points (a)-(d) in the discussion that follows Eq. (2a).

Perhaps of interest, when $n \approx 0$ one may replace $\partial_{2} U_{2}$ with $Z_{\mathrm{p}} U_{2}$ in Eq. (12c), which provides an alternative expression that confirms the findings in Example 5 in [1].

In sum, it appears that the theoretically-derived expressions Eqs. (12a)-(12c) represents a Step 2-model which has a capability of modelling deformation wear.

\subsection{Impact Wear}

Consider a transient liquid impact erosion process, starting at time $t=0$. Study the process at $t>0$ following a collapsed gas cavity in a liquid-gas flow, or an impacting liquid in a gas-liquid flow. On the fluid side of the target surface, in the vicinity of a fixed target surface position, the gaseous phase (or so-called "voidage" in the field of multiphase flow), which was present just prior to impact at $t<0$, disappears at $t=0$, and is replaced by a liquid phase which exerts mechanical work transfer in the form of shock work. Beyond a certain threshold level, an irreversible indentation- or fracture process occurs, resulting in impact wear, $c f$. Fig. 5 in [1].

It is a widely-accepted experimental result that the timeintegrated energy of reflecting waves (which can be estimated using an acoustic recorder, located at a distance from the target surface) is often proportional to the net impact wear, when studying cavitation erosion. (This experimental correlation is arguably only observed at conditions above associated threshold levels.)

The theoretically-derived impact wear expression Eq. (31) in [1], when time-integrated, can also be correlated with the time-integrated energy of reflecting (or throughpropagating) waves, when assuming the impact wear work 
to be directly proportional to the shock wave work (i.e. neglecting the influence of thresholds):

$$
\begin{aligned}
& -\int \dot{w}_{\text {pressuresurface, irr }} \mathrm{d} t=-\int \alpha\left(P_{\text {proc }}-P_{\text {noproc }}\right) \nabla \cdot \mathbf{U d} t \\
& \propto\left[\alpha=\alpha_{\mathrm{f}}=1\right] \propto-\int P \nabla \cdot \mathbf{U d} t \\
& =\left[\begin{array}{l}
\text { transient equation of continuity: } \\
\frac{\partial \rho}{\partial t}=-\nabla \cdot(\rho \mathbf{U}) \cong-\rho \nabla \cdot \mathbf{U}
\end{array}\right] \cong \int \frac{P}{\rho} \mathrm{d} \rho \\
& =\left[\left.\frac{\mathrm{d} P}{\mathrm{~d} \rho}\right|_{s, \rho=\rho_{0}} \stackrel{\text { Def. }}{=} c^{2} \gg 1 \Rightarrow \mathrm{d} P \gg \mathrm{d} \rho\right] \cong \frac{1}{\rho} \int P \mathrm{~d} \rho \\
& =\left[\mathrm{d} \rho=\frac{\mathrm{d} P}{c^{2}}\right]=\frac{1}{\rho c^{2}} \int_{0}^{\Delta P} P \mathrm{~d} P=\frac{(\Delta P)^{2}}{2 \rho c^{2}} \equiv \varepsilon_{\mathrm{pot}}
\end{aligned}
$$

where $\rho$ is the fluid density, $c$ is the speed of sound in the fluid, and $\varepsilon_{\text {pot }}$ is the potential energy density of the shock wave. Hence, the time-integrated net impact wear work in Eq. (31) in [1] is proportional to the time-integrated energy of the reflecting shock waves, i.e. proportional to the impact wear, in agreement with experimental results. In order to obtain a model coefficient, and associated threshold, the author believes that one must necessarily perform correlating experiments.

\section{Conclusions}

Different aspects on connecting the theoreticallyderived differential expressions of wear work or attrition work, to corresponding net wear or net attrition, have been presented and discussed.

It was found necessary that one consider suitable and relevant boundary conditions, in order to achieve correct modelling of net wear or net attrition.

At extremum conditions, it may be required to fine-tune some approximate nominal flow models, such as boundary conditions, in order to follow basic thermodynamic requirements. Arguably, this should be done without altering the basic structure of the process mechanisms.

The assumed direct proportionality between sub-process wear/attrition work and sub-process outcome (net wear/attrition) is demonstrated for several cases. With the present approach, overall modelling of attrition in a TMP refiner is significantly improved. Also, directly from a wear work mechanism, one may compute ideal Finnie erosion, Archard abrasion, and also explain the particle-size dependency behavior on ductile wear. Also, a theoreticallyderived impact wear differential mechanism will - when time-integrating across an impact wear process - result in net wear which is directly proportional to the potential energy density of reflecting waves. This is a correlation which agrees with experimental observations.

Furthermore, an important role of kinetic theory was found - implicating a dependence on particle dynamics. If experimental behavior is not known, one may utilize kinetic theory to roughly estimate the behavior of particle viscosity, or the slip-flow parameter.

Analysis of ductile attrition in a TMP wood chip refiner shows that attrition occurs spot-wise by necessity, and hence not in a continuum manner.

\section{Acknowledgements}

This work was supported by Hot Disk AB (Sweden). Special thanks to D.Sc. S.E. Gustafsson at Dept. Physics, Chalmers Univ. of Technology, Dr. H. Otterberg at University of Gothenburg, Assoc. Prof. J. Gustavsson and Assoc. Prof. Å. Haglund at Dept. Microtechnology \& Nanoscience, Chalmers Univ. of Technology, as well as Dr. Daniel Cederkrantz and Dr. Ma Yi at Hot Disk AB (Sweden), for assistance in preparing this manuscript.

\section{Nomenclature}

A

$c$

C

$C_{1}$

$C_{2}$

$C_{3}$

$C_{4}$

$C_{5}$

$C_{A}, C_{B}, C_{C}$,

$C_{D}, C_{E}, C_{F}$

$\mathrm{CS}$, wall

$\mathrm{CV}$, spots

$d_{p}$

E

$F_{\mathrm{F}}$

$F_{\mathrm{N}}$

a

$H$

$K^{\text {strong }}$

$K^{\text {weak }}$

L

$L_{\text {ang }}$

$L_{\text {nom }}$

$m$

$m_{\text {ductileerosion }}$

$n$

n

$N$

$P$

$r$ gross contacting area $\left(\mathrm{m}^{2}\right)$ speed of sound in fluid $\left(\mathrm{m} \mathrm{s}^{-1}\right)$ general model constant model constant defined in Eq. (1d) $\left(\mathrm{kg} \mathrm{m}^{-1} \mathrm{~s}^{-2}\right)$ model constant defined in Eq. (4) $\left(\mathrm{kg} \mathrm{m}^{-1} \mathrm{~s}^{-2}\right)$ model constant defined in Eq. (2a) $\left(\mathrm{kg}^{-1} \mathrm{~m}^{2} \mathrm{~s}^{2}\right)$ model constant defined in Eq. (B1) $\left(\mathrm{s}^{2} \mathrm{~m}^{-2}\right)$ model constant defined in Eq. (B2) $\left(\mathrm{s} \mathrm{m}^{-1}\right)$

non-dimensional model constants wall side of control volume surface local control volume where discrete breakage/deformation of particles occurs mean particle diameter, or mean diameter of abrasives ( $\mathrm{m}$ ) energy $(\mathrm{J})$ tangential force applied by abrasives across a gross contacting area $A(\mathrm{~N})$ normal force applied by abrasives across

gross contacting area $A(\mathrm{~N})$

hardness $\left(\mathrm{N} \mathrm{m}^{-2}\right)$

wear coefficient, defined by $K^{\text {strong }}=C_{1} H \chi / C_{2}\left(\mathrm{~N} \mathrm{~m}^{-2}\right)$

wear coefficient used in relation between total work and wear rate, defined in Eq. (A1) (-)

slip flow coefficient $(\mathrm{m})$

correction coefficient of $L_{\text {nom }}(-)$

nominal slip flow coefficient ( $\mathrm{m}$ )

mass ( $\mathrm{kg}$ )

ductile erosion target surface mass loss per impacted particle $(\mathrm{kg})$

real number variable $(-)$

surface unit normal vector $(-)$

number of particles per second $\left(\mathrm{s}^{-1}\right)$

static pressure $\left(\mathrm{N} \mathrm{m}^{-2}\right)$

radial position in TMP refiner $(\mathrm{m})$ 


\section{Greek letters}

$\alpha$

$\alpha_{k}$

$\Phi$

$\delta_{\mathrm{NCVD}}$ slid distance in Archard model ( $\mathrm{m}$ )

time ( $\mathrm{s}$ )

Cartesian component $i$ of mean velocity vector of phase $k\left(\mathrm{~m} \mathrm{~s}^{-1}\right)$ mean velocity vector of phase $k\left(\mathrm{~m} \mathrm{~s}^{-1}\right)$ mean (slip) velocity vector of phase $k$ at the surface $\left(\mathrm{m} \mathrm{s}^{-1}\right)$

particle nominal impact velocity $\left(\mathrm{m} \mathrm{s}^{-1}\right)$

slip velocity at the surface $\left(\mathrm{m} \mathrm{s}^{-1}\right)$

wear volume $\left(\mathrm{m}^{3}\right)$

work rate transferred at surface

$\left(\mathrm{W} / \mathrm{m}^{2}\right)$, or work rate exerted by fluid element $\left(\mathrm{W} / \mathrm{m}^{3}\right)$

defibration work rate $\left(\mathrm{W} / \mathrm{m}^{3}\right)$

ductile wear work rate $\left(\mathrm{W} / \mathrm{m}^{2}\right)$

ductile wear work rate ( W )

non-specified type of work rate $\left(\mathrm{W} / \mathrm{m}^{2}\right)$

non-specified type of work rate ( W )

wall friction work rate $\left(\mathrm{W} / \mathrm{m}^{2}\right)$

wall friction work rate ( W )

other types of irreversible work rate

$\left(\mathrm{W} / \mathrm{m}^{2}\right)$

other types of irreversible work rate ( W )

irreversible pressure work rate transferred at surface $\left(\mathrm{W} / \mathrm{m}^{2}\right)$

irreversible work rate transferred at surface $\left(\mathrm{W} / \mathrm{m}^{2}\right)$

shock wave transfer/reflection work rate $\left(\mathrm{W} / \mathrm{m}^{2}\right)$

shock wave transfer/reflection work rate ( W ) real number

Cartesian co-ordinates ( $\mathrm{m}$ )

model coefficient

particle motion pre-impact angle (-) average occurrence of phase $k(-)$ gap width in 2-body or 3-body abrasion experiment, or minimum local gap width in TMP refining process $(\mathrm{m})$ normal components of the viscous dissipation term for the particulate phase $\left(\mathrm{W} \mathrm{m}^{-3}\right), c f$. definition in [5]

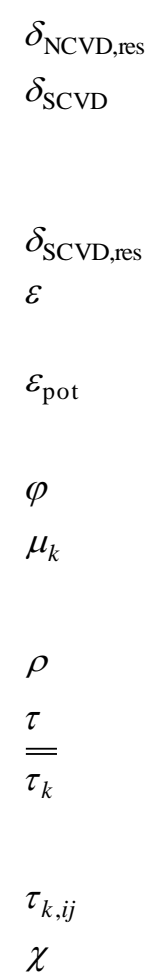

\section{Special notations}

$\Delta(\cdot) \quad$ difference

$\nabla($.$) \quad gradient$

$\mathrm{d}(\cdot) \quad$ differential

$(\cdot)$ isentropic conditions

$(\cdot)_{\text {surface }} \quad$ conditions in vicinity of surface

$\partial_{1}, \partial_{2} \quad$ differentiation $\frac{\partial}{\partial y_{1}}, \frac{\partial}{\partial y_{2}}$

$\mathrm{D}(\cdot) / \mathrm{D} t$

substantial derivative [e.g. following the scratcher position in an Archard experiment (Lagrangian view), which can be re-expressed in terms of fixed-wear position (Eulerian view) on target surface following the relation $\mathrm{D}(\cdot) / \mathrm{D} t \equiv \partial(\cdot) / \partial t+(\mathbf{U} \cdot \nabla)(\cdot)$ 
Appendix A: Empirically-Obtained Archard Relation Reformulated Into an Eulerian Expression

The empirical Archard relation [17-19] $V=K^{\text {weak }} F_{\mathrm{N}} s / H$, is analyzed for ductile materials, where $F_{\mathrm{N}}$ is the normal force applied by the abrading particles (or contact point for 2-body abrasion) across a gross contacting area $A$ (the forces in play on the net contacting area are not known), $K^{\text {weak }}$ is an empirical (non-dimensional) abrasive wear coefficient, $S$ is the sliding distance, $H$ is the hardness of the abraded (target surface) material, and $V$ is the abraded wear volume.

This weak relation, a Lagrangian expression, can be reformulated into an Eulerian expression for a fixed targetsurface position, correlating wear rate proportional to total work rate:

$$
\begin{aligned}
& \underbrace{\text { wear rate }[\mathrm{m} / \mathrm{s}]}_{\text {at fix position }}=\frac{\mathrm{d}}{\mathrm{d} t}\left(\frac{V}{A}\right)=\frac{\mathrm{D}}{\mathrm{D} t}\left(\frac{K^{\text {weak }} F_{\mathrm{N}} s}{A H}\right) \\
& \text { continuous } \\
& \text { flow } \\
& \stackrel{\substack{\text { assumption } \\
=}}{\frac{\partial}{\partial t}\left(\frac{K^{\text {weak }} F_{\mathrm{N}} s}{A H}\right)}+\mathbf{U} \cdot \nabla\left(\frac{K^{\text {weak }} F_{\mathrm{N}} s}{A H}\right) \\
& =\frac{K^{\text {weak }} F_{\mathrm{N}}}{A H} U_{\text {slip }}=\left[F_{\mathrm{F}} \stackrel{\text { Def. }}{=} \chi F_{\mathrm{N}}\right] \\
& \frac{K^{\text {weak }}}{H} \frac{F_{\mathrm{F}}}{A} \frac{U_{\text {slip }}}{\chi}=\frac{K^{\text {weak }}}{H \chi} \tau U_{\text {slip }} \\
& =\frac{K^{\text {weak }}}{H \chi} \cdot\left(\text { total work rate }\left[\mathrm{W} / \mathrm{m}^{2}\right]\right)
\end{aligned}
$$

The coefficient $\chi$ represents the ratio of tangentialversus normal force acting on the gross contacting area.

Earlier, a strong relation between wear rate and wear work was argued for, $c f$. Eq. (4). Inserting Eq. (1d) into Eq. (4), one obtains:

$$
\begin{aligned}
& \underbrace{\text { wear rate }[\mathrm{m} / \mathrm{s}]}_{\text {at fix position }}=\frac{1}{C_{2}} \cdot \underbrace{\left(\text { wear work rate }\left[\mathrm{W} / \mathrm{m}^{2}\right]\right)}_{=\left.C_{1} L \delta_{\mathrm{SCVD}, \text { res }}\right|_{\text {surface }}} \\
& =\left.\left(\frac{C_{1}}{C_{2}}\right) L \delta_{\mathrm{SCVD}, \text { res }}\right|_{\text {surface }}
\end{aligned}
$$

One may assign $C_{1} / C_{2}=K^{\text {strong }} / H \chi$, where $H$ and $\chi$ are identically same numbers as in Eq. (A1), which renders the non-dimensional wear coefficient $K^{\text {strong }}$ (numerically different than $K^{\text {weak }}$ ).

Hence, one obtains the following strong relation:

$$
\underbrace{\text { wear rate }[\mathrm{m} / \mathrm{s}]}_{\text {at fix position }}=\left.\frac{K^{\text {strong }} L}{H \chi} \cdot \delta_{\text {SCVD,res }}\right|_{\text {surface }}
$$

Since the wear rate in Eq. (A1) is identical to the wear rate in Eq. (A2), one may directly associate the original Archard relation to the wear work through the following expression:
$\frac{\mathrm{D}}{\mathrm{D} t}\left(\frac{K^{\text {weak }} F_{\mathrm{N}} s}{A H}\right)=\left.\frac{K^{\text {strong }} L}{H \chi} \cdot \delta_{\mathrm{SCVD}, \mathrm{res}}\right|_{\text {surface }}$

Arguably, 2-body and 3-body abrasion can be directly connected with the wear work rate.

\section{Appendix B: Newtonian/Lagrangian Finnie Single- Particle Ductile Erosion Model}

The Finnie single-particle ductile erosion model [2] is a model, which albeit being derived employing a Newtonian/Lagrangian approach, presents the net integrated erosion at a fixed position (vicinity of the impact point) on the target surface, in terms of a mass loss of the ductile target surface per impacted particle:

$$
m_{\text {ductileerosion }}=C_{4} m_{p} U_{\text {n.i.v. }}^{2} f c t(\alpha) \quad[\mathrm{kg} / \text { particle }]
$$

where $0 \leq f c t(\alpha) \leq 1$ describes the angular behavior of the net erosion. Hence, since Eq. (B1) already presents the wear at a fixed position, there is no need to make any dynamic transformation from the Lagrangian framework to the Eulerian framework. However, in order to make a valid comparison of Eq. (B1) with the general Eulerian model Eq. (2b), it is required that a corresponding Eulerian representation of Eq. (B1) should be compared with Eq. (2b) (when applying a representative and corresponding flow as model input for Eq. (2b)).

Equation (B1) can for this purpose be reformulated in terms of a target surface erosion rate for a steady rain-type flow of impacting particles. To proceed, consider a Finnie experiment being performed in a confined volume $V_{\text {region }}$, for erosion of an inclined plate having a surface area $A_{\text {surface }}$, with a rate of $N$ particles per second (the particles do not interact with each other), impacting at velocity $U_{\text {n.i.v. }}$ with impact angle $\alpha$. For this experimental configuration, one can set $m_{p} \propto \rho_{p} d_{p}{ }^{3}$ and $\alpha_{p} \propto N d_{p}{ }^{3}$. The volume removal rate per particle is $V_{\text {surface }}=m_{\text {ductileerosion }} / \rho_{\text {surface }}$. An Eulerian erosion-rate version of the Finnie single-particle erosion model can then be outlined as follows:

erosion rate $[\mathrm{m} / \mathrm{s}]=\frac{\mathrm{d}}{\mathrm{d} t}\left(\frac{V_{\text {surface }}}{A_{\text {surface }}}\right)$

$=\frac{N C_{4} m_{p} U_{\text {n.i.v. }}{ }^{2} f c t(\alpha)}{A_{\text {surface }} \rho_{\text {surface }}}$

$=C_{5}\left(\frac{\rho_{p}}{\rho_{\text {surface }}}\right) \alpha_{p} U_{\text {n.i.v. }}^{2} f c t(\alpha)$

The Eulerian Finnie-erosion rate model Eq. (B2) applies for a rain-type erosive impact over the entire target surface, with no particles interacting with any other particles during the process. Also, reflecting particles do not interact with incoming particles.

In order to compare the general model Eq. (2b) with the Eulerian representation of the Finnie model Eq. (B2), it is necessary to obtain an Eulerian representation of the specific flow conditions assumed by Finnie, to be applied in Eq. (2b): In the vicinity of the wall, a chipping motion [2] 
of a protruding tip of the impacting particle into a ductile material is in the Eulerian framework comparable to a sum of two flow components: a contribution from the inflow as well as a contribution from the reflecting flow:

$$
\left.U_{p, \mathrm{t}}\right|_{\text {surface }} \cong \frac{1}{2}(1+b(\alpha)) U_{\text {n.i.v. }} \cos \alpha
$$

The component $0 \leq b(\alpha)<1$ accounts for the reflection flow contribution to the averaged slip-flow velocity at the wall, taking into account the exhaustion of the tangential particle velocity due to the tangential-direction cuttingmotion kinetic energy loss. Finnie observed an erosion maximum around $17^{0}$ for many materials, which provides information on $b(\alpha)$. Finnie assumed complete exhaustion for impact angles above (approximately) the erosion maximum impact angle, which means that $b(\alpha) \cong 0$ when $\alpha>17^{0}$, i.e. corresponding to a diffuse reflection of particles for $\alpha>17^{0}$. To obtain the case $L \rightarrow \infty$ at $0^{0}$ impact [according to Observation (B)], it is necessary to set $b\left(\alpha=0^{0}\right) \cong 1$. According to Finnie, between $0^{0}$ and $17^{0}$ the particle cuts into the ductile material - the tip travelling in a parabolic manner, cutting through the target surface - and the particle leaves the target surface with a not completely exhausted kinetic energy. Consequently, the particle leaves the target surface with a tangential velocity magnitude statistically greater than zero. A crude model, in order to study the angular behavior of $L$, can be obtained by approximating $b(\alpha) \cong\left(17^{0}-\alpha\right) / 17^{0}$ for $0^{0}<\alpha<17^{0}$. Inserting Eq. (B3) into Eq. (2b) (and utilizing Eqs. (5)-(6)), one obtains the following result from the general model Eq. (2b):

$$
\begin{aligned}
& \text { erosion rate }[\mathrm{m} / \mathrm{s}] \cong \\
& \overbrace{K^{\text {strong }}}^{\text {term A }}[\overbrace{\left(\frac{\alpha_{p}\left(\mu_{p, \text { proc }}-\mu_{p, \text { noproc }}\right)}{4 L_{\text {nom }}}\right)}^{\text {term B }} U_{\text {n.i.v. }}^{2} \\
& \underbrace{\left(C_{1}\right)^{-1} \times \text { wear work }}_{\times\left\{\frac{((1+b(\alpha)) \cos \alpha)^{2}}{L_{\text {ang }}(\alpha)}\right\}}]
\end{aligned}
$$

where $L(\alpha)=L_{\text {nom }} L_{\text {ang }}(\alpha)$. For different impact angles, one may assume that term A is constant, following the assumption on a directly proportional correlation between wear work and net wear rate, $c f$. Eq. (4). From this, arguably $K^{\text {strong }}$ is constant for different impact angles following Eq. (4) and Finnie's own assumption of a constant $\chi$ (i.e. constant ratio of normal to tangential forces acting on cutting tips of abrasives) in single-particle erosion processes (irrespective of impact angle and whether exhaustion of the particle's kinetic energy has occurred or not) - an assumption which provided Finnie with excellent agreement with corresponding experiments. Comparing Eq. (B4) with Eq. (B2), one can note that the only component which influences angular dependency requires $((1+b(\alpha)) \cos \alpha)^{2} / L_{\text {ang }}(\alpha)=f c t(\alpha)$ from which $L_{\text {ang }}(\alpha)$ can be determined through experiments, or directly calculated from the ideal Finnie model or similar model. It can be shown that $L_{\text {ang }}(\alpha)$ follows Observations (A) and (B), when inserting the $f c t(\alpha)$-curve (which is obtained in experiments or from the Finnie model), $c f$. Fig. B1. It can be noted that for a ductile material having a different angle of maximum erosion, a modified $b(\alpha)$ for Eq. (B3) can be implemented.
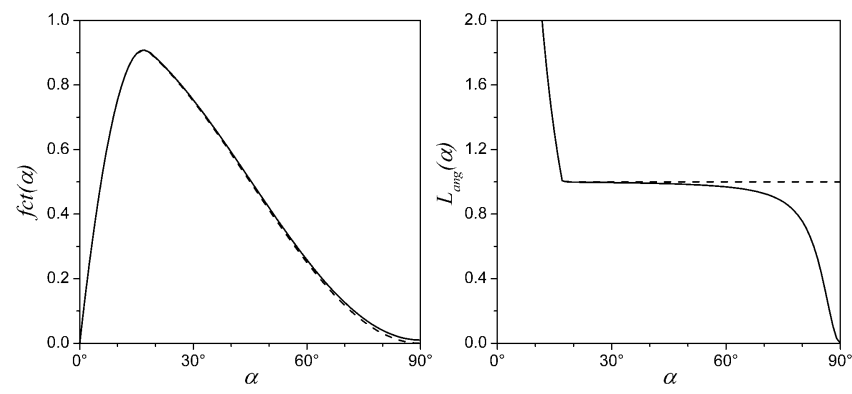

Figure B1. Plot of $f c t(\alpha)$ representing Finnie model for single-particle impact erosion of ductile materials (left), and corresponding plot of $L_{a n g}(\alpha)$ (right). The ideal Finnie model (hashed line) can be represented by $f c t(\alpha)=3\left(\sin 2 \alpha-3 \sin ^{2} \alpha\right)$ for $0<\alpha<\alpha_{0}$ and $f c t(\alpha)=\cos ^{2} \alpha$ for $\alpha_{0}<\alpha<90^{\circ}$, where $\alpha_{0}=18.43^{\circ}$. According to the literature, the ideal Finnie model works well for small angles of attack, but underestimates ductile erosion for larger angles. Several models are proposed in the literature to improve modelling of ductile erosion at larger angles of attack. The full line (left) represents a small correction of the ideal Finnie model according to $f c t(\alpha)=\cos ^{2} \alpha+0.01 \sin ^{1.5} \alpha$ for $\alpha_{0}<\alpha<90^{\circ}$, and corresponding plot (right) of $L_{\text {ang }}(\alpha)$. Any ductile erosion angular dependency, for single-particle or any dense-particle jet streams, has a fct $(\alpha)$ for which a corresponding $L_{\text {ang }}(\alpha)$ can be plotted. The general ductile wear model Eq. (2b) models ductile erosion with identical results to the ideal or adjusted Finnie model, when the $L_{\text {ang }}(\alpha)$ curves are known. It should also be noted that Observations (A) and (B) apply, with the exception of the ideal Finnie model at higher angles of impact, where $L_{a n g}(\alpha)=1$. A correction of the ideal Finnie model, as well as experimental results for erosive flows, indicate that $L_{\text {ang }}(\alpha) \rightarrow 0$ when $\alpha \rightarrow 90^{\circ}$.

Hence, one obtains $L_{\text {ang }}(\alpha)$ which applied in Eq. (B4) provides a model which applies for most single-particle ductile erosion situations.

Comparing Eq. (B4) (which originates from the general model Eq. (2b)) to a comparable erosion-rate expression of the Finnie model, Eq. (B2), allows us to equate the coefficients:

$C_{5}\left(\frac{\rho_{p}}{\rho_{\text {surface }}}\right)=\frac{K^{\text {strong }}}{4 H \chi L_{\text {nom }}}\left(\mu_{p, \text { proc }}-\mu_{p, \text { noproc }}\right)$

Hence, the general ductile erosion model Eq. (2b) also has the capability of modelling single-particle ductile erosion.

\section{Appendix C: Size Effect on Abrasion and Erosion of Ductile Materials}

It is believed possible to estimate the size-effect by conducting an order-of-magnitude analysis of Eq. (1e), assuming a directly proportional correlation between ductile wear work and net ductile wear rate.

Consider the geometrical constraints in the abrasion experiments illustrating the size effect. In the literature are presented $\mathrm{x}$-y-graphs, in which experimentally-recorded, and normalized, wear is presented on y-axis, vs. particle size as on $\mathrm{X}$-axis.

Arguably the gap width in an abrasion experiment is of crucial importance - a gap width that provides a connection with the involved (bounded) flow gradients. Since the gap width is not reported in the relevant experiments in 
literature (since experimental apparatus are not designed to maintain constant gap when testing different-size abrasives), a simple assumption on the correlation between size of the abrasive and gap width can be assumed, based on assumptions on the operation of the experimental apparatus utilized. [Note: A fundamental $1^{\text {st }}$-law requirement is that a flow gradient needs to be considered for the case of 2-body abrasion. Arguably, a fair measure of the flow gradient is to consider the relative velocity between cutter and target surface, $U$, divided by the gap width, $\Phi$, i.e. $\partial U / \partial y \sim U / \Phi$, as a relation representing the flow gradient. This assumption is utilized below.]

For the case of erosion, where a gap width cannot be defined, instead the (bounded) flow gradients are of concern. As shown below, the behavior of flow gradients differ if the erosive particle flow is dilute, or dense. These differences arguably influence the apparent normalized size effect on erosion.

Other geometrical constraints involved would be the influence of possible surface roughness, and shape of the abrasives.

For erosion and 3-body abrasion, one may consider an influence on the surface slip-velocity.

If conducting an order-of-magnitude analysis, whereby a bounded flow gradient is expressed in terms of the other above, highlighted parameters (following the geometrical assumptions illustrated in Fig. 1 in [1]), arguably the following flow gradients can be roughly estimated:

For 2-body and 3-body abrasion, with a gap width of $\Phi$, the flow gradient can be expressed as:

flow gradient $=U /(\Phi+\varepsilon)=U /\left(d_{p}+\varepsilon\right)$

$=C_{E} U /\left(C_{B} d_{p}+\varepsilon\right)$

(2-body)

or

flow gradient $=U /(\Phi+\varepsilon)$

$=\left(U-2 U_{\text {slip }}\right) /\left(C_{B} d_{p}+\varepsilon\right)$

$=C_{E} U /\left(C_{B} d_{p}+\varepsilon\right)$

(3-body)

where $C_{B}=1$ and $C_{E}=1$ for 2-body abrasion, and $C_{B}>1$ and $0<C_{E}\left(d_{p}\right)<1$ for 3-body abrasion. The coefficient $C_{E}$ may for 3-body abrasion display practically fixed value with varying particle size. Alternatively, the coefficient $C_{E}$ may for 3-body abrasion decrease slightly with increasing particle size, indicating an increase in relative slip velocity with increasing particle size. [Note: the fundamental requirement of bounded flow gradients, and also non-zero fluid element, also in the limit $d_{p} \rightarrow 0$, requires adjustment of the nominal gradient expression. The introduction of a small $\varepsilon$, of the order 1-10 microns, is one approach.]

In case of erosion, there are two different behaviors. Within a small distance from the target surface, say $\Phi=5 d_{p}$, a tangential component of the flow, directly associated with the nominal impact velocity (and impact angle), can be identified as $U=C_{A} U_{\text {n.i.v. }}$, where $0<C_{A}<1$ . It can be noted that reducing the particle size will not adjust these relationships. Also, it is clear from Fig. C1, that the flow velocity in the vicinity of the target surface, $U_{p, t}$, directly associates with $U$, such that $U_{p, t}=C_{C} U$, where $0<C_{C}<C_{A}<1$. We get:

flow gradient $=\left(U-U_{p, t}\right) /(\Phi+\varepsilon)$

$=U\left(1-C_{C}\right) /\left(C_{B} d_{p}+\varepsilon\right)$

$=C_{E} U /\left(C_{B} d_{p}+\varepsilon\right) \quad$ (dilute erosion)

where $C_{B}>1$ and $0<C_{E}<1$ for dilute erosion. The implications that follows the Finnie theory is that the coefficient $C_{C}$ (and in turn $C_{E}$ ) should not vary with varying particle sizes. The existence of a flow gradient in the case of dilute erosion is obvious, since this flow gradient must fundamentally exist (according to thermodynamics) in order for an irreversible wear work transfer to at all be possible.
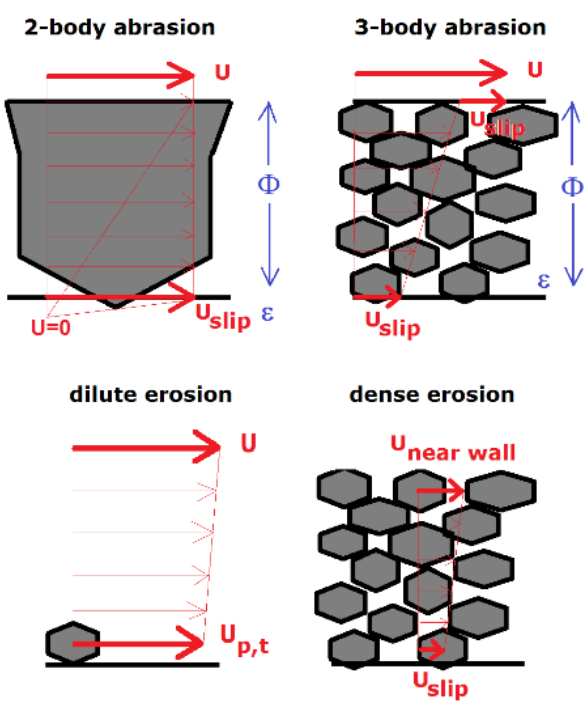

Figure C1. Illustrating the assumption on flow gradient. Following the continuum assumption applied, a continuous velocity profile (without any discrete jumps) is modelled.

A different situation is at hand for the case of denseflow ductile erosion. Near the wall, for small particle diameters, the flow velocity is small. For the situation that $d_{p} \rightarrow 0$, it can be shown that $U_{\text {slip }} \rightarrow 0$. (For continuum flows of fluids representing gases or liquids, the no-slip condition assumption applies, i.e. effectively assuming that $U_{\text {slip }}=0$ at solid walls.) A normalization can hence not be made against any fixed far-from-surface external flow velocity (such as that controlling the experiment). If a similar linear approximation is made within a small distance $\Phi=5 d_{p}$ from the target surface, we may express:

$$
\begin{aligned}
& \text { flow gradient }=\left(U_{\text {near wall }}-U_{\text {slip }}\right) /(\Phi+\varepsilon) \\
& =U_{\text {slip }}\left(C_{D}-1\right) /\left(C_{B} d_{p}+\varepsilon\right) \\
& =C_{F} d_{p} /\left(C_{B} d_{p}+\varepsilon\right) \quad \text { (dense erosion) }
\end{aligned}
$$

where $C_{B}>1, C_{D}>1$ and $C_{F}>0$ are constants.

Inserting these order-of-magnitude flow gradient estimations into Eq. (1e), one may compute corresponding ductile wear rate versus abrasive size $d_{p}$. In Figs. C2-C3 are normalized graphs of the computed ductile wear rates, 
for a couple of different situations (assuming $\varepsilon$ equal to 5 micrometers).

Interestingly, the present order-of-magnitude analysis provide a direct ability of computing - in a straightforward manner - normalized curves which fully match the available experimental curves on particle-size dependency presented in the literature. Earlier, this was not possible, since the size-effect behavior has not earlier been possible to explain.

It can be noted that some experimental curves show erosion rate maximum at a certain particle size (such as found in 3-body abrasive wear experiments). This erosion maximum situation is possible to reproduce with present order-of-magnitude analysis, $c f$. green curve in Fig. C2, where the explanation for this behavior is simply that $U_{\text {slip }}$ is not directly proportional to $U-$ a not uncommon situation according to the sciences of multiphase flows.

This order-of-magnitude analysis applies at far-fromequilibrium conditions, above the ductile wear onset threshold.

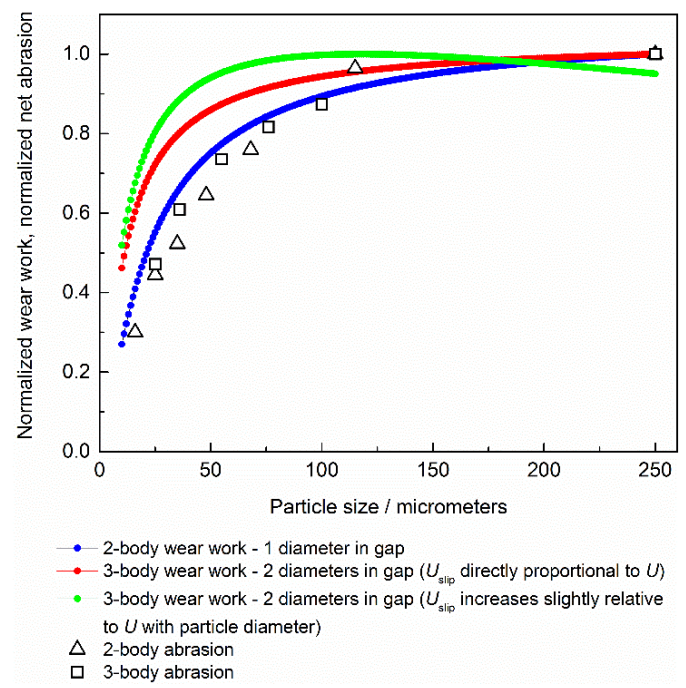

Figure C2. Particle-size dependency on ductile abrasion and ductile wear work (for an abrasive flow), normalized. Experimental data taken from [8] (originally presented in [7]). The experimental data points represent "wear rate as a function of SiC particle size for copper [...] in two body and three-body abrasion (volume removed/(load $x$ distance travelled))". Ductile wear work is analytically computed.

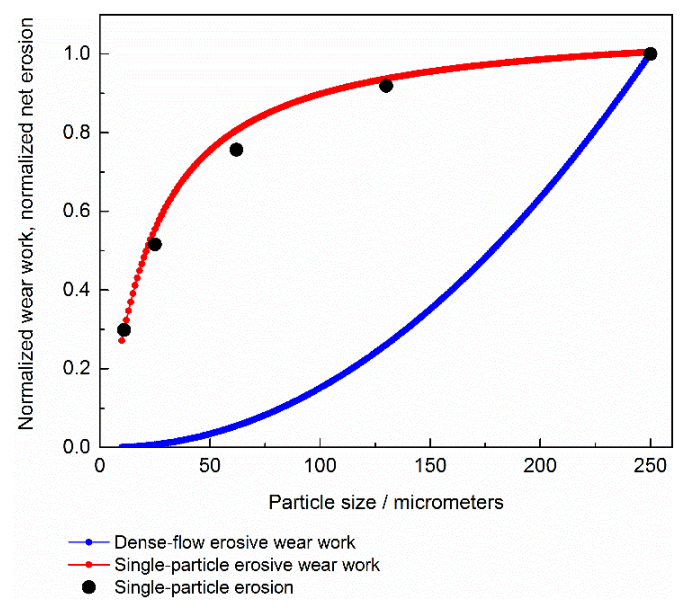

Figure C3. Particle-size dependency on ductile erosion and ductile wear work (for an erosive particle flow), normalized. Experimental data taken from [8] (originally presented in [7]). The experimental data points represent "wear rate as a function of $\mathrm{SiC}$ particle size for copper in erosion (mass loss/mass of abrasive)". Ductile wear work is analytically computed.

\section{References:}

[1] M. Gustavsson, "'A residual thermodynamic analysis of inert wear and attrition, Part 1: Theory', Int. J. Thermodyamics, 18, 27-38, 2015.

[2] I. Finnie, "Erosion of surfaces by solid particles", Wear, 3, 87-103, 1960.

[3] M. Gustavsson, A. E. Almstedt, 'Two-fluid modelling of cooling-tube erosion in a fluidized bed', Chem. Eng. Sci., 55, 867-879, 2000.

[4] R. W. Lyczkowski, S. Folga, S. L. Chang, J. X. Bouillard, C. S. Wang, G. F. Berry, D. Gidaspow, "State-of-the-art computation of dynamics and erosion in fluidized bed tube banks', in: Proceedings of the $10^{\text {th }}$ International Conference on Fluidized Bed Combustion, Vol. 1, ASME, New York, 465-478, 1989.

[5] M. Gustavsson, "Fluid dynamic mechanisms of particle flow causing ductile and brittle erosion", Wear, 252, 845-858, 2002.

[6] M. Gustavsson, "Residual thermodynamics: A framework for analysis of non-linear irreversible processes", Int. J. Thermodynamics, 15, 69-82, 2012.

[7] A. Misra, I. Finnie, "On the size effect in abrasive and erosive wear, Wear, 65, 359-373, 1981.

[8] I. Finnie, "Some reflections on the past and future of erosion', Wear, 186-187, 1-10, 1995.

[9] S. Chapman, T. G. Cowling, The Mathematical Theory of Non-Uniform Gases; An Account of the Kinetic Theory of Viscosity, Thermal Conduction and Diffusion in Gases, $3^{\text {rd }}$ ed., Cambridge University Press, Cambridge, New York, 1991.

[10] J. Ding, R. W. Lyczkowski, S. W. Burge, D. Gidaspow, "Three-dimensional models of hydrodynamics and erosion in fluidized-bed combustors', AIChE Symp. Ser., 88, 85-98, 1992.

[11] I. Finnie, Y. H. Kabil, "On the formation of surface ripples due to erosion', Wear, 8, 60-69, 1965.

[12]D. Berg, A. Karlström, M. Gustavsson, "Deterministic consistency estimation in refining processes", in: International mechanical pulping conference, PAPTAC, Montreal, Canada, 361-366, 2003.

[13] K. Eriksson, An Entropy-based Modeling Approach to Internally Interconnected TMP Refining Processes, (Licentiate dissertation). Chalmers University of Technology, Gothenburg, Sweden, 2005.

[14] A. Karlström, D. Sikter, M. Gustavsson, “Plate gap estimation based on physical refining measurements", in: International Mechanical Pulping Conference, TAPPI, Minneapolis, Minnesota, USA, 2007.

[15] A. Karlström, K. Eriksson, D. Sikter, M. Gustavsson, "Refining models for control purposes", Nord. Pulp Paper Res., 23, 129-138, 2008.

[16] J. Kestin, A Course in Thermodynamics, vol. I, New York: Taylor \& Francis, 1979. 
[17] J. F. Archard, "Contact and rubbing of flat surfaces", J. Appl. Phys., 24, 981-988, 1953.

[18]E. Rabinowicz, Friction and Wear of Materials, New York: Wiley, 1965.
[19] J. F. Archard, W. Hirst, "The Wear of metals under unlubricated conditions", in: Proceedings of the Royal Society of London. Series A, Mathematical and Physical Sciences, 236, 397-410, 1956. 